\title{
Multicriteria Supplier Classification for DSS: Comparative Analysis of Two Methods
}

\author{
J.M. Sepulveda, I.S. Derpich
}

\author{
Juan M. Sepulveda*, Ivan S. Derpich \\ University of Santiago \\ 3769 Ecuador Ave., Santiago, Chile, CP 7254758 \\ *Corresponding author: juan.sepulveda@usach.cl \\ ivan.derpich@usach.cl
}

\begin{abstract}
In this paper the analysis of two multicriteria decision making (MCDM) methods for sorting suppliers in industrial environments is presented. The MCDM methods correspond to Electre and FlowSort and both are applied to the classification of providers in an actual case of the local softdrink bottling industry in Chile. The results show that Electre as an outranking method it may well classify suppliers in a similar manner as FlowSort does. Nevertheless, due to the intrinsic underlying fuzzy multicriteria nature of the problem, FlowSort is found to be more suitable method for building a rule-based system based on preference functions for automating the process of suppliers clustering when developing strategies of relationship management in the sense of the Kraljic categories in supply chain management.
\end{abstract}

Keywords: Decision Support Systems, Supply Management, Electre, FlowSort.

\section{Introduction}

Supplier management is crucial in order to improve the benefits that a company can have at the operational, functional, economic, and financial levels and in terms of the supply chain it is embedded. Supplier evaluation is a regular process of operational management in many companies; the need arises from finding suppliers for new products, parts, or materials, or for assessing performance of the current supplier base in order to decide continuation of their services. However, in supplier management a more strategic task is classifying suppliers into categories for deciding the relational approach to be followed. Kraljic [1] in his pioneering work established categories of suppliers according to the economic impact for the purchasing company and the risks the suppliers may experiment in their respective market. As recently reviewed by Monczka et. al. [2] Kraljic's categories are defined by a matrix of four quadrants, namely: routine, leverage, bottleneck and critical quadrant. Each category means different strategies ranging from automated transactions by the use of ERP and/or EDI of commodity items of low total purchasing expenses, i.e. routine category, up to the critical category in which for example the expenses are very high and the suppliers situation in its market is risky due to uncertainties, uniqueness or high dependence. See Figure 1 for an example of the Kraljic matrix. The number in parenthesis is a level in a scale from 1 to 4 , as explained below.

Level 1: Many alternative products and processes; abundant sources of supply; low valuesmall individual transactions; routinary use, unspecified items; no specialized purchasing knowledge required.

Level 2: Complex specifications requiring complex manufacturing or service processes; few alternate production/sources of supply; big impact on operations / maintenance; new technology or untested processes.

Level 3: High expenditures, commodity items; large marketplace capacity, big inventories; many alternate products and services; many qualified providers; market/price sensitive demand.

Level 4: Critical to profitability and operations; few qualified sources of supply; large expenditures; design and quality critical; complex and/or rigid specifications. 


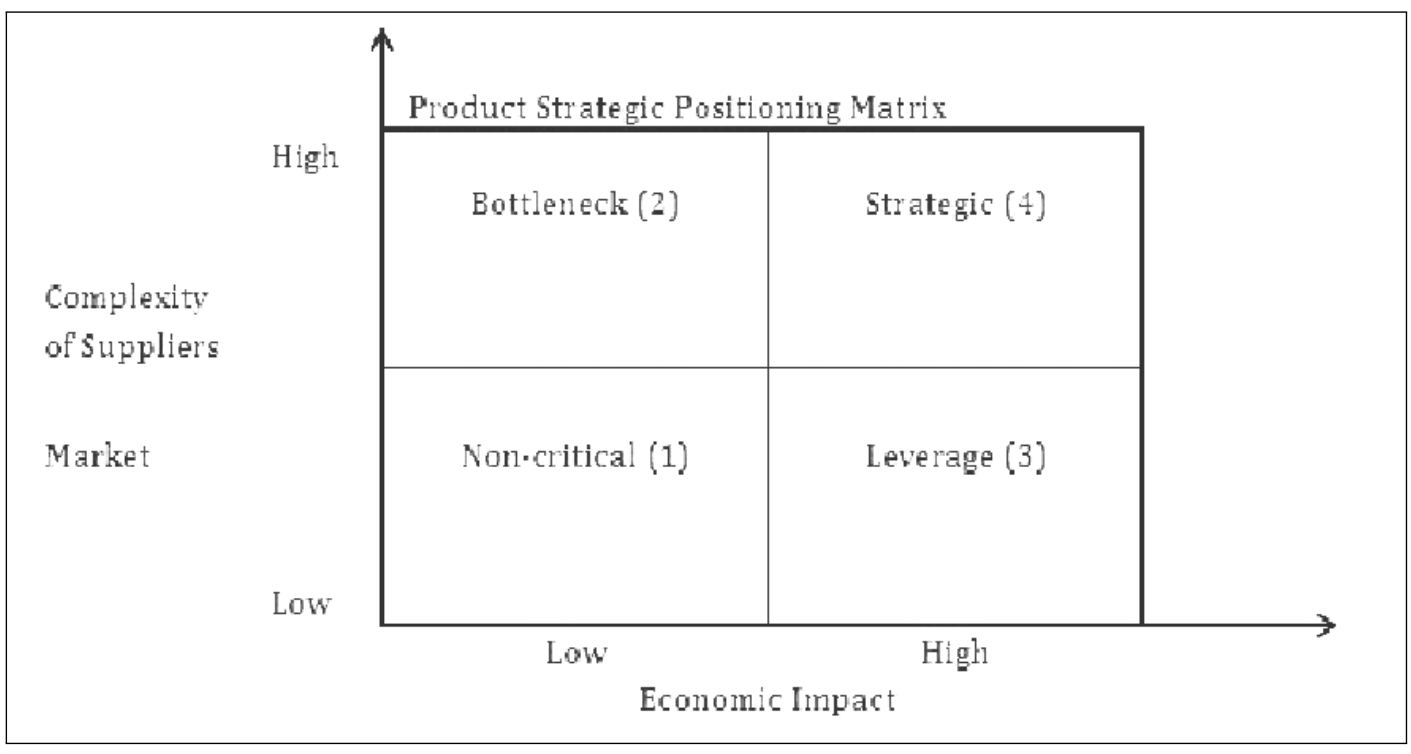

Figure 1: Kraljic Matrix 1 for strategic positioning

In this work the classification or categorization of suppliers in the case study, defines three categories: (a) transactional, (b) collaborative, and (c) integrated. These categories are specific for the company dealt with; however the method is general enough as to be applied to other similar situations.

A transactional supplier is understood as the profile of routine suppliers combining low purchasing expenses, easy-to-obtain commodity-type products or services, and with low mutual attraction in the relationship. A collaborative supplier is one of importance to the company due to high purchasing expenses. For this reason, close monitoring of performance is needed in order to assure the service level agreements and to control the costs. The logistics complexity is of medium range as well as the mutual attraction. An integrated supplier is a critical one, of such an importance that a strategic alliance is needed, such as the case of a third party fully manufacturing a component or a product for a given customer company's brand (OEM strategy), as it occurs in the food, pharmaceutical, or car industries. It combines criticality in product positioning with high logistics complexity and high mutual attraction of the relationship.

Clearly, the three categories above can be understood as fuzzy sets where the implementation process of the method allows the identification of strengths and weaknesses of using formalized supplier selection models to tackle the supplier sorting problem. This highlights potential barriers preventing the adoption of these types of methods. For this purpose, the paper presents a number of relevant issues arising from the application and managerial implications for both customer and suppliers, concerning differentiated management practices according to their classification.

Besides Matrix 1, two other matrices are used to classify suppliers. Matrix 2 values the complexity of logistics, and Matrix 3 the mutual attraction of the SRM (supplier relationship management).

In Matrix 2 (Lead Time and Stock Rotation) the lowest level of logistics complexity is for supplies of fast replenishment and slow moving items. It the follows the case of suppliers of items with short lead time (hours, days, within a week) but of high consumption or inventory turnover. The third level is for slow moving items and long lead times (e.g. imported products); finally, the most complex logistics is for suppliers of items with high rotation and long lead time due the risk of inventory breakdown.

The numbers assigned as well as the criteria are depending on the specific application and are 


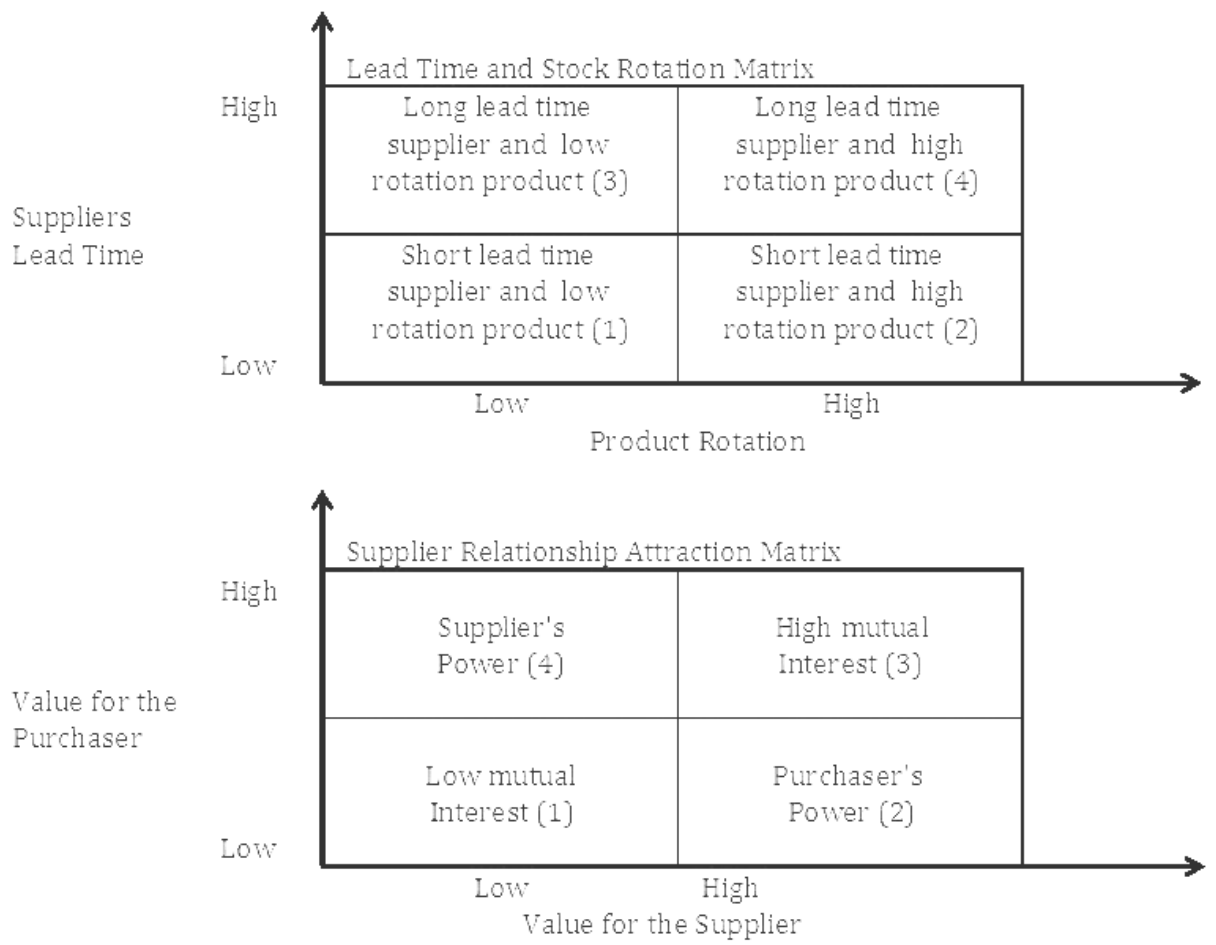

Figure 2: Matrices 2 and 3 for logistics complexity and SRM attraction level

provided by experienced purchasing executives, without loss of generality. In Matrix 3 (Supplier Relationship Attraction) the lowest level is assigned when both parties have little mutual interest. It follows when the purchaser is important to the supplier (level 2). For the specific case of the application, the managers gave the highest importance to suppliers with superior power in the relationship (generally due to size).

\section{Strategies by category}

With the suppliers being categorized, the organization can apply differentiated approaches to SRM. Next, a brief description of each category is given.

\subsection{Transactional category}

The transactional category has as main characteristics the existence of many alternative products and services, many sources of supply, low value small individual transactions, commoditytype items, requiring little or no specialization of the purchasing executive. Hence, this category is non-critical and besides the logistics aspects are simple because lead times are short and rotation is low. The mutual relationship is of low level. The general strategy for this category is the simplification of the acquisition process. The tactics relate to increase the role of computer based systems and reducing the buying effort. Specific actions include rationalization of the supplier base, the automation of the purchasing process by the use of automated requisitioning, electronic data interchange, stockless procurement, minimization of administrative costs. Little negotiation is needed. 


\subsection{Collaborative category}

The collaborative category has as main characteristics the existence of many alternative products and services, many sources of supply, commodity-type items, requiring little or no specialization of the purchasing executive. However, the purchasing expenses and volumes are higher. Hence, the optimization of operations serve as leverage for obtaining commercial advantage. Logistics may be more complex due to longer lead times and higher rotation of items. The type of relationship is of purchaser's power. The general strategy for this category is maximizing the commercial advantage. The tactics involve concentration of purchasing to increase power and also to maintain competition. Specific actions involve the promotion of competitive bidding, procurement coordination, adoption of industry standards to reduce dependency.

\subsection{Integrated category}

This category has as main characteristics a heavy control of the providers performance, partnerships in the form of a strategic outsourcing, cooperation to optimize mutual benefits. For instance, a strategic supplier performing outsourced implant critical tasks of production and operations. The complexity of operations demands close control and information exchange. The importance of the purchaser and the criticality of the supplier calls for relations of mutual benefits. The attraction force of both parties is high.

\section{Basics of the two methods}

The ELECTRE methodology is based on the concordance and discordance indices. The simplest method of the ELECTRE family is ELECTRE-I. See Figueira et al. [3].

This method starts from the data in the decision matrix, and assumes that the sum of the weights of all criteria is equal to one. For an ordered pair of alternatives $\left(A_{j}, A_{k}\right)$, the concordance index $c_{j k}$ is the sum of all the weights for those criteria where the performance score of $A_{j}$ is least as high as that of $A_{k}$, as in formula (1). It can be seen that $c_{j k}$ lies between 0 and 1.

$$
c_{j k}=\sum_{i: a_{i j} \geq a_{i k}} w_{i} \quad j, k=1, . ., n, j \neq k
$$

The computation of the discordance index $d_{j k}$ is more elaborated: $d_{j k}=0$ if $a_{i j}>a_{i k}$, $i=1, \ldots, m$, that is, the discordance index is zero if $A_{j}$ performs better than $A_{k}$ on all of the criteria. Otherwise, $d_{j k}$ is calculated as in (2). That is, for each criterion where $A_{k}$ outperforms $A_{j}$, the ratio is calculated as the difference in performance level between $A_{k}$ and $A_{j}$ and the maximum difference in score on the criterion concerned between any pair of alternatives.

$$
d_{j k}=\max _{i=1, . ., m} \frac{a_{i k}-a_{i j}}{\max _{j=1, . ., n} a_{i j}-\min _{j=1, \ldots, n} a_{i j}} \quad j, k=1, . ., n, j \neq k
$$

The maximum of these ratios (which must lie between 0 and 1 ) is the discordance index. A concordance threshold $c^{*}$ and discordance threshold $d^{*}$ are then defined such that $0<d^{*}<c^{*}<$ 1. Then, $A_{j}$ outranks $A_{k}$ if the $c_{j k}>c^{*}$ and $d_{j k}<d^{*}$, i.e. the concordance index is above and the discordance index is below its threshold, respectively.

FlowSort is based on the Promethee ranking methodology (Figueira et al., 2005) [3], this new sorting method by Nemery and Lamboray [4] is utilized for assigning actions to completely ordered categories; these categories are defined either by limiting profiles (i.e., min and max values) or by central profiles (or centroids). This method has also been applied by (Sepulveda 
et al., 2010) [5] in another management decision problem in the innovation field for diagnosing capabilities in small enterprises.

In what follows, limiting profiles will be used. The assignment of an action (i.e., an object to be sorted) into a category is based on the relative position of this action with respect to the defined reference profiles in terms or incoming or outgoing net flows. Let $A=\left(a_{1}, a_{2}, \ldots, a_{n}\right)$ be the set of $n$ actions or alternatives to be sorted. These actions are evaluated on $q$ criteria $g_{j}(j=1, \ldots, q)$; all criteria are supposed to be maximized in the decision making problem. The categories to which the actions must be assigned are denoted by $C_{1}, C_{2}, \ldots, C_{k}$. Let $R=\left(r_{i}, \ldots, r_{K+1}\right)$ be the set of limiting profiles in the case when a category is defined by an upper and lower limit. Let $\pi(x, y)$ be the preference of an action $x$ over an action $y$, as in the Promethee method.

Figure 3 shows typical shapes of preference functions where the $x$-axis is the degree of difference between actions $x$ and $y$. Thus, the positive, negative and net flows $\phi$ of each action $x$ in $R$, are computed by equations (4) (5) (6) where $\dot{R}_{i}=R \mathrm{U}\left\{a_{i}\right\}$ is the extended set of profiles either for the limiting profile case or the central profiles. The rules for assigning actions $a_{i}$ to a category $C_{h}$ are given by equations (7) and (8) in the case of limiting profiles.

$$
\begin{gathered}
\pi(x, y)=\sum_{j=1}^{q} w_{j} P(x, y) \\
\phi_{\dot{R}_{i}}^{+}=\frac{1}{\left|\dot{R}_{i}\right|-1} \sum_{y \in \dot{R}_{i}^{+}} \pi(x, y) \\
\phi_{\dot{R}_{i}}^{-}=\frac{1}{\left|\dot{R}_{i}\right|-1} \sum_{y \in \dot{R}_{i}^{-}} \pi(x, y) \\
\phi_{\dot{R}_{i}}=\phi_{\dot{R}_{i}}^{+}-\phi_{\dot{R}_{i}}^{-} \\
C_{\phi^{+}}\left(a_{i}\right)=C_{h}, \quad \text { if } \quad \phi_{\dot{R}_{i}}^{+}\left(r_{h}\right) \geq \phi_{\dot{R}_{i}}^{+}\left(a_{1}\right)>\phi_{\dot{R}_{i}}^{+} \\
C_{\phi^{-}}\left(a_{i}\right)=C_{h}, \quad \text { if } \quad \phi_{\dot{R}_{i}}^{-}\left(r_{h}\right)<\phi_{\dot{R}_{i}}^{-}\left(a_{1}\right) \leq \phi_{\dot{R}_{i}}^{-}\left(r_{h+1}\right)
\end{gathered}
$$

\section{Results of the two methods}

First, the sorting will be made by the Electre method. Table 1 shows the score combination for six randomly chosen suppliers and the resulting category according to the values predefined for the combination by using Electre. The six cases are for illustrative purposes and show the type of results without loss of generality.

Table 2 shows the limiting profiles for the FlowSort method.

The scores for each criteria in FlowSort are the same as the combination values in Table 1.

Table 3 shows the results for FlowSort. In the table, the six extended sets $\mathrm{R}$ correspond to suppliers A,B,C,D,E,F, respectively. The category is obtained by applying the rules defined by equations (7) and (8). It can be observed that for the six suppliers, with the exception of $\mathrm{C}$, the assigned categories are the same.

This is encouraging since the basis of each method is very different. It can be said that in case $\mathrm{C}$ this provider was better classified by FlowSort. The difference obtained in one of the suppliers (Supplier C), is mainly because of the assessment data that show that this provider 


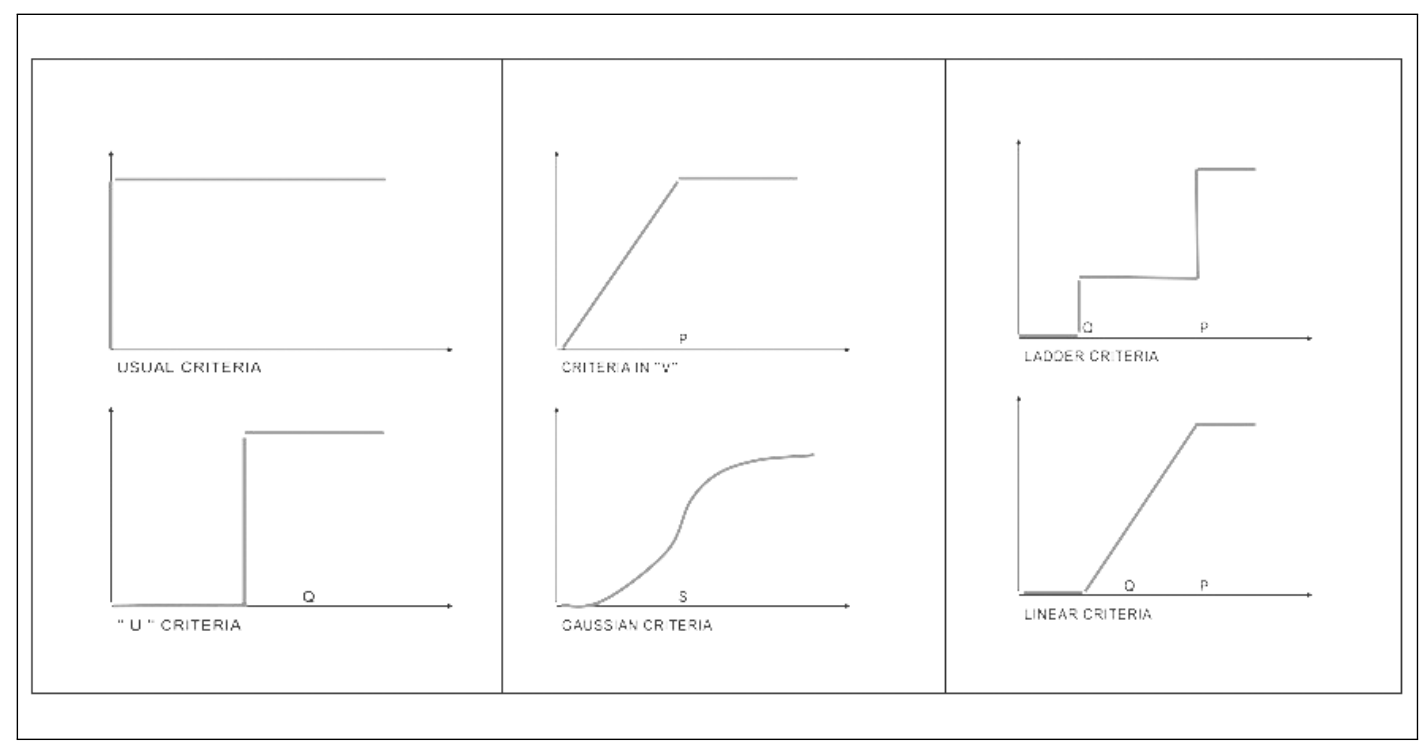

Figure 3: Types of Preference functions

Table 1: Results by Electre

\begin{tabular}{|c|c|c|}
\hline Supplier & Score combination & Category \\
\hline A & "4-3-3" & Integrated \\
\hline B & "3-2-2" & Collaborative \\
\hline C & "1-2-3" & Transactional \\
\hline D & "4-3-4" & Integrated \\
\hline E & "4-3-4" & Integrated \\
\hline F & "4-3-2" & Integrated \\
\hline
\end{tabular}

Table 2: Limiting profiles chosen for FlowSort

\begin{tabular}{|c|c|c|c|}
\hline Profile & $C_{1}$ & $C_{2}$ & $C_{3}$ \\
\hline$r_{1}$ & 4.5 & 4.5 & 4.5 \\
\hline$r_{2}$ & 3.0 & 3.0 & 3.0 \\
\hline$r_{3}$ & 1.5 & 3.0 & 3.0 \\
\hline$r_{4}$ & 0 & 0 & 0 \\
\hline
\end{tabular}




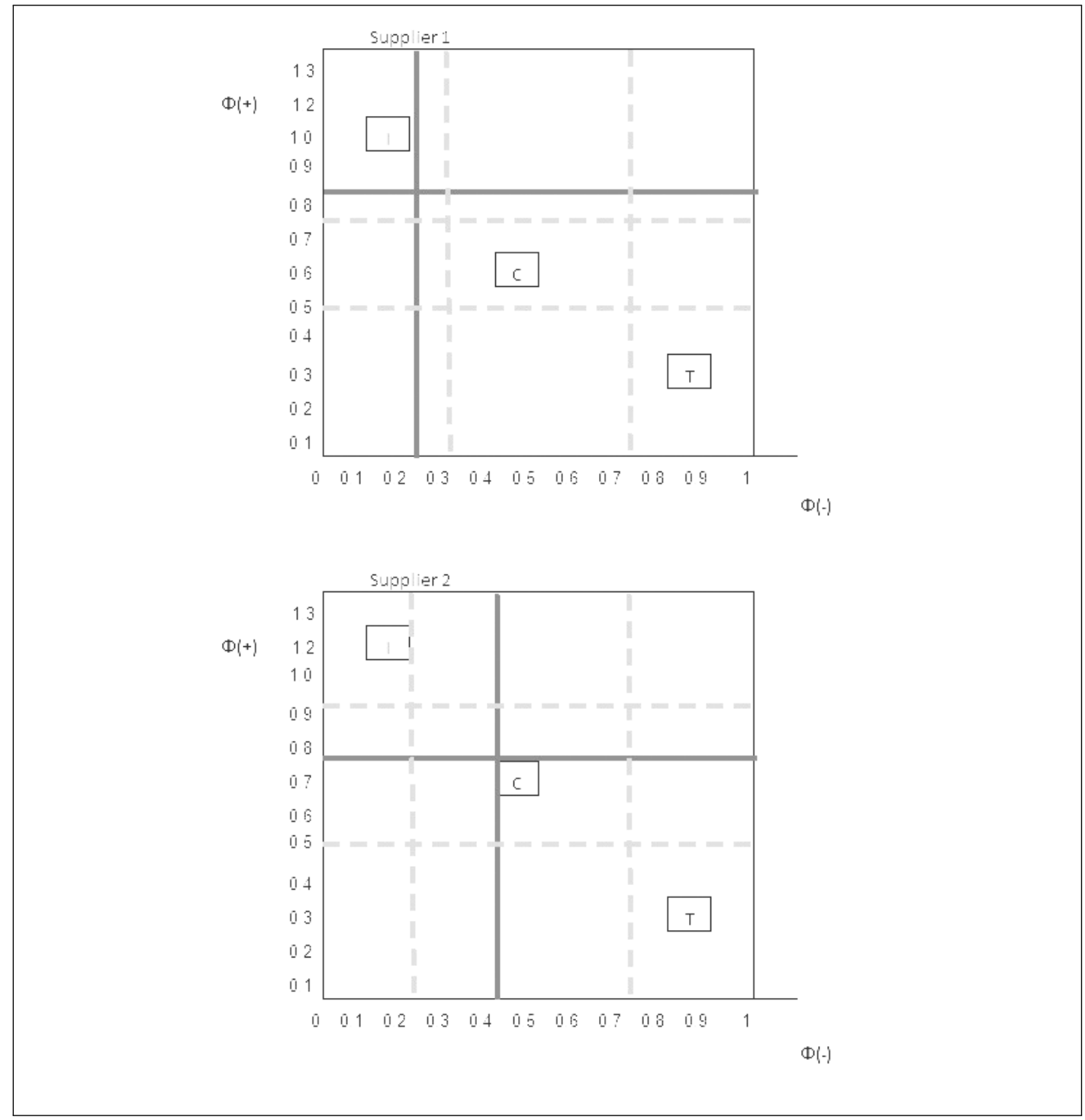

Figure 4: Graphical Representation of FlowSort Results for Two Suppliers 
Table 3: Results for FlowSort

\begin{tabular}{|c|c|c|c|c|c|c|c|}
\hline Flow Sort & Results & $r_{1}$ & $r_{2}$ & $r_{3}$ & $r_{4}$ & $A(i)$ & Category \\
\hline \multirow{3}{*}{1} & $\phi_{+}$ & 1.25 & 0.75 & 0.5 & 0 & 0.83 & Integrated \\
& $\phi_{-}$ & 0 & 0.33 & 0.75 & 1 & 0.25 & Integrated \\
& $\phi_{\text {net }}$ & 1.25 & 0.42 & -0.25 & -1 & 0.58 & Integrated \\
\hline \multirow{2}{*}{2} & $\phi_{+}$ & 1.25 & 0.92 & 0.5 & 0 & 0.75 & Collaborative \\
& $\phi_{-}$ & 0 & 0.25 & 0.75 & 1 & 0.42 & Collaborative \\
& $\phi_{\text {net }}$ & 1.25 & 0.67 & -0.25 & -1 & 0.33 & Collaborative \\
\hline \multirow{2}{*}{3} & $\phi^{+}$ & 1.25 & 0.92 & 0.58 & 0 & 0.67 & Collaborative \\
& $\phi^{-}$ & 0 & 0.25 & 0.67 & 1 & 0.5 & Collaborative \\
& $\phi^{\text {net }}$ & 1.25 & 0.67 & -0.08 & -1 & 0.17 & Collaborative \\
\hline \multirow{R}{*}{4} & $\phi^{+}$ & 1.25 & 0.75 & 0.5 & 0 & 0.92 & Integrated \\
& $\phi^{-}$ & 0 & 0.42 & 0.75 & 1 & 0.25 & Integrated \\
& $\phi^{\text {net }}$ & 1.25 & 0.33 & -0.25 & -1 & 0.67 & Integrated \\
\hline \multirow{2}{*}{5} & $\phi^{+}$ & 1.25 & 0.75 & 0.5 & 0 & 0.92 & Integrated \\
& $\phi^{-}$ & 0 & 0.42 & 0.75 & 1 & 0.25 & Integrated \\
& $\phi^{\text {net }}$ & 1.25 & 0.33 & -0.25 & -1 & 0.67 & Integrated \\
\hline \multirow{2}{*}{6} & $\phi^{+}$ & 1.25 & 0.83 & 0.5 & 0 & 0.83 & Integrated \\
& $\phi^{-}$ & 0 & 0.33 & 0.75 & 1 & 0.33 & Integrated \\
& $\phi^{\text {net }}$ & 1.25 & 0.5 & -0.25 & -1 & 0.5 & Integrated \\
\hline
\end{tabular}

has some lower scores in some criteria (matrix- criterion 1) and high in other ones ( 2 and 3 ) and the final class depends on the decision maker.

However, note that FlowSort is a method that reflects the in and out flows generated by the alternatives, while the classification made in the Electre is arbitrary at some extent by the threshold values chosen for each category. Nevertheless, both methods solve the problem and are suitable for automating the classification process as part of a decision support system for supply management.

Figure 4 shows a graphical representation of the sorting for Suppliers 1 and 2, as given by Table 3.

In Table 1 the category is chosen according to the values in Table 4 . This shows the results of the Electre method for the 64 possible combinations of the three matrices with the aggregated dominance index (concordance minus discordance). The score values of Table 4 in columns Score Matrix 1,2,3 (indicated as SM1, SM2, SM3) for each supplier score combination (SC) were given by experienced supply managers same as in the FlowSort method. Current work is addressing the obtention of these numbers from the key performance indicators in the database of a business intelligence module connected to the ERP system.

The categories are obtained by sorting in increasing order of the aggregated dominance index (A.D.I.) and by defining thresholds for each category. The weights were chosen as $1 / 3$ each matrix (w.l.g). For instance, as shown in Table 1, Supplier A with scores "4-3-3" corresponds to the combination "a60"in the Appendix, Supplier B with scores "3-2-2" corresponds to "a35", and so on. In this case, the same cases were used for both the Electre and FlowSort methods. 
Table 4: Categories by Electre

\begin{tabular}{|c|c|c|c|c|c|c|c|c|c|c|c|}
\hline $\mathrm{SC}$ & SM1 & SM2 & SM3 & A.D.I. & Category & $\mathrm{SC}$ & SM1 & SM2 & SM3 & A.D.I. & Category \\
\hline a1 & 1 & 1 & 1 & 0 & Transactional & a34 & 3 & 1 & 4 & 17 & Collab. \\
\hline a2 & 1 & 1 & 2 & 1 & Transactional & a37 & 4 & 1 & 3 & 17 & Collab. \\
\hline a3 & 1 & 2 & 1 & 1 & Transactional & a38 & 4 & 3 & 1 & 17 & Collab. \\
\hline a4 & 2 & 1 & 1 & 1 & Transactional & a30 & 1 & 3 & 4 & 18 & Collab. \\
\hline a5 & 1 & 1 & 3 & 2 & Transactional & a31 & 1 & 4 & 3 & 18 & Collab. \\
\hline a6 & 1 & 3 & 1 & 2 & Transactional & a36 & 3 & 4 & 1 & 18 & Collab. \\
\hline a7 & 3 & 1 & 1 & 2 & Transactional & a 40 & 2 & 2 & 4 & 20 & Integrated \\
\hline a8 & 1 & 1 & 4 & 3 & Transactional & a43 & 4 & 2 & 2 & 20 & Integrated \\
\hline a9 & 1 & 2 & 2 & 3 & Transactional & a41 & 2 & 4 & 2 & 21 & Integrated \\
\hline a10 & 1 & 4 & 1 & 3 & Transactional & a46 & 3 & 2 & 3 & 21 & Integrated \\
\hline a11 & 2 & 1 & 2 & 3 & Transactional & a 47 & 3 & 3 & 2 & 21 & Integrated \\
\hline a12 & 2 & 2 & 1 & 3 & Transactional & a45 & 2 & 3 & 3 & 22 & Integrated \\
\hline a13 & 4 & 1 & 1 & 3 & Transactional & a44 & 4 & 4 & 1 & 27 & Integrated \\
\hline a14 & 1 & 2 & 3 & 6 & Transactional & a39 & 1 & 4 & 4 & 28 & Integrated \\
\hline a15 & 1 & 3 & 2 & 6 & Transactional & a 42 & 4 & 1 & 4 & 28 & Integrated \\
\hline a16 & 2 & 1 & 3 & 6 & Transactional & a 50 & 3 & 2 & 4 & 31 & Integrated \\
\hline a17 & 2 & 3 & 1 & 6 & Transactional & a 53 & 4 & 3 & 2 & 31 & Integrated \\
\hline a18 & 3 & 1 & 2 & 6 & Transactional & a48 & 2 & 3 & 4 & 32 & Integrated \\
\hline a19 & 3 & 2 & 1 & 6 & Transactional & a49 & 2 & 4 & 3 & 32 & Integrated \\
\hline a23 & 2 & 2 & 2 & 7 & Collaborative & a 51 & 3 & 4 & 2 & 32 & Integrated \\
\hline $\mathrm{a} 20$ & 1 & 2 & 4 & 10 & Collaborative & a 52 & 4 & 2 & 3 & 32 & Integrated \\
\hline $\mathrm{a} 21$ & 1 & 4 & 2 & 10 & Collaborative & a 54 & 3 & 3 & 3 & 35 & Integrated \\
\hline a 22 & 2 & 1 & 4 & 10 & Collaborative & a 56 & 4 & 2 & 4 & 43 & Integrated \\
\hline a24 & 2 & 4 & 1 & 10 & Collaborative & a 57 & 4 & 4 & 2 & 43 & Integrated \\
\hline a25 & 4 & 1 & 2 & 10 & Collaborative & a 55 & 2 & 4 & 4 & 44 & Integrated \\
\hline a26 & 4 & 2 & 1 & 10 & Collaborative & a58 & 3 & 3 & 4 & 45 & Integrated \\
\hline $\mathrm{a} 27$ & 1 & 3 & 3 & 11 & Collaborative & a 59 & 3 & 4 & 3 & 45 & Integrated \\
\hline a28 & 3 & 1 & 3 & 11 & Collaborative & a60 & 4 & 3 & 3 & 45 & Integrated \\
\hline a29 & 3 & 3 & 1 & 11 & Collaborative & a61 & 3 & 4 & 4 & 55 & Integrated \\
\hline a32 & 2 & 2 & 3 & 12 & Collaborative & a62 & 4 & 3 & 4 & 55 & Integrated \\
\hline a33 & 2 & 3 & 2 & 12 & Collaborative & a63 & 4 & 4 & 3 & 55 & Integrated \\
\hline a 35 & 3 & 2 & 2 & 13 & Collaborative & a64 & 4 & 4 & 4 & 63 & Integrated \\
\hline
\end{tabular}

\section{Conclusions and future works}

In this article, the comparison of two methods for supplier sorting for determining the supplier management strategy in large organizations has been presented. Assigning a category to a supplier is an important task within supply chain management since many types of suppliers are commonly in place and differentiated management approaches are needed in order to accomplish the efficiency and service objectives. The sorted categories combined three main dimensions: strategic positioning (matrix 1), logistics complexity (matrix 2), and attraction of the mutual relation (matrix 3). While the original Kraljic's matrix is concerned only with strategic positioning, giving four categories of suppliers: non-critical or routine, leverage, bottleneck, and critical, the contribution in this work is that the analysis has been extended to other dimensions, such as logistics complexity (matrix 2) and the attraction of mutual relation (matrix 3).

By using the extended Kraljic's matrix concept developed in this work, a portfolio of strategies may be identified according to the defined categories: transactional, collaborative, and integrated suppliers. Commonly, the analysis of suppliers is performed based on experienced managers over a restricted number of cases. In large organizations, however, because of the high number of suppliers, such manual method becomes difficult and prone to error. With multicriteria sorting 
models it is possible to overcome this weakness and even automate this task. Flowsort was compared to ELECTRE. A good coincidence was obtained between these two methods. However Electre requires previously defined categories by using explicit enumeration and threshold values entered manually by experts. In this sense, Flowsort requires less human input being more adaptable for automated processing.

As direction for future works, further research is needed in order to examine the robustness of the results and the effects of the scales used in the assessment. Also, in order to minimize human data input, the scores in the matrices ideally should be obtained in a direct manner from the key performance indicators (KPI) obtained from the ERP system of the organization, or from a business intelligence (BI) module, among other aspects. Ongoing work of the authors is addressing these issues.

\section{Acknowledgments}

The authors are very grateful to DICYT (Scientific and Tecnological Research Office), Project Number 061117SS and the Industrial Engineering (IE) Department, both of the University of Santiago of Chile for their support in this work. Also to IE graduates Marcos Melin and Stephanie Sepulveda who helped in the data collection and model implementation.

\section{Bibliography}

[1] Kraljic, P. (1983); Purchasing must become supply management, Harvard Business Review, September-October, 1983.

[2] Monczka, R.M., Handfield, R.B., Giunipero, K.C., Patterson, J.L. (2011); Purchasing And Supply Chain Management, 5th Edition, Cengage Learning.

[3] Figueira, J., Greco S., Ehrgott M. (2005); Multiple Criteria Decision Analysis: State of the Art Surveys, Springer-Verlag.

[4] Nemery P.; Lamboray C. (2008); FlowSort: a flow-based sorting method with limiting or central profiles, TOP, 16, 90-113, Springer-Verlag.

[5] Sepulveda, J., Gonzalez, J., Alfaro, M. (2010); A Metrics-based Diagnosis Tool for Enhancing Innovation Capabilities in SMEs, International Journal of Computers Communications E Control, 5(5):919-928. 